\title{
Diabetes remission off medications is not a suitable endpoint for comparing bariatric/metabolic surgery with pharmacotherapy
}

\author{
Bruno Halpern $^{1,2}$ - Cintia Cercato ${ }^{1,2,3}$ - Marcio C. Mancini ${ }^{1,2,3}$
}

Received: 14 April 2016 / Accepted: 15 April 2016 / Published online: 16 June 2016

(C) Springer-Verlag Berlin Heidelberg 2016

Keywords Bariatric surgery · Diabetes pharmacotherapy · Diabetes relapse · Diabetes remission · Metabolic surgery · Obesity $\cdot$ Roux-en-Y gastric bypass $\cdot$ Sleeve gastrectomy . Type 2 diabetes · Weight loss

$\begin{array}{ll}\text { Abbreviations } & \\ \text { ILMI } & \text { Intensive lifestyle and medical intervention } \\ \text { RYGB } & \text { Roux-en-Y gastric bypass } \\ \text { STAMPEDE } & \text { Surgical Treatment and Medications } \\ & \text { Potentially Eradicate Diabetes Efficiently }\end{array}$

To the Editor: In their publication in Diabetologia, Cummings et al [1] describe how they used a randomised trial to compare the best available intensive lifestyle and medical intervention (ILMI) against Roux-en-Y gastric bypass (RYGB), and concluded that surgery achieved greater type 2 diabetes remission in mildly to moderately obese patients. However, mean $\mathrm{HbA}_{1 \mathrm{c}}$ values were not statistically different between groups at the end of study, which was not explicitly stated in the abstract. An OR for diabetes remission (defined as $\mathrm{HbA}_{1 \mathrm{c}}$ $<6.0 \%[<42.1 \mathrm{mmol} / \mathrm{mol}]$, off all diabetes medicines) of

Bruno Halpern

brunohalpern@hotmail.com

1 Obesity Unit, Department of Endocrinology, Hospital das Clínicas, University of São Paulo-Brazil (USP), São Paulo, Brazil

2 Brazilian Association for the Study of Obesity and Metabolic Syndrome (ABESO), Rua Mato Grosso 306/1711, CEP 01239-040 São Paulo, SP, Brazil

3 Obesity Department of Brazilian Society of Endocrinology and Metabolism (SBEM), Rio de Janeiro, Brazil almost 20 looks quite impressive, but in our opinion this is not a suitable study endpoint for analysis. Given that the comparison group received intensive lifestyle with optimal medical intervention, and therefore the very point of the intervention was the use of medication, it seems inappropriate that a criterion of the primary endpoint of interest was whether or not the patient was off medication. It is well established that diabetes is a chronic disease and most patients are unable to control their diabetes with intensive lifestyle interventions (the same applies to obesity itself, despite being associated with greater and persistent stigma). Therefore, the discontinuation of several classes of medications for type 2 diabetes would give rise to disease deterioration. As expected with any other chronic disease, the control group could be well controlled, but, of course, on medication. Since a surgical procedure can be considered a chronic treatment for diabetes (because the anatomical changes are permanent), this seems to be an unfair comparison. We believe that a better judgment (which still would favour surgery in the great majority of cases) would be an $\mathrm{HbA}_{1 \mathrm{c}}$ target that was independent of medication use. We acknowledge that the criteria for remission were not developed solely for this article and are being used in most bariatric/metabolic surgery studies, but because of the conclusion of the abstract and the absence of statistically significant differences in $\mathrm{HbA}_{1 \mathrm{c}}$ at the end of the treatment, this comment seems appropriate at this point.

Also of note is the conclusion about individuals with a BMI $<35 \mathrm{~kg} / \mathrm{m}^{2}$. It is stated in the Results section that 'In exploratory analyses, diabetes remission was not predicted by baseline BMI, age or sex, or by the amount of weight lost during 1 year, and there was no correlation between change in body weight and change in $\mathrm{HbA}_{1 \mathrm{c}}$ at 6 or 12 months among those having RYGB; however, the study was not specifically powered to detect this.' Furthermore, in the Discussion section the authors state that 'there is still limited evidence from RCTs 
examining surgical approaches to type 2 diabetes treatment in patients with a BMI $<35 \mathrm{~kg} / \mathrm{m}^{2}$, the standard threshold for bariatric surgery, and our study adds Level-1 data to that evidence base'. We would like to emphasise that no more than five patients with a BMI $<35 \mathrm{~kg} / \mathrm{m}^{2}$ underwent surgery in this study, an obviously undersized sample from which to make inferences about this population separately. In fact, in RCTs performed to date, fewer than 150 diabetic patients with a BMI $<35 \mathrm{~kg} / \mathrm{m}^{2}$ have been studied, making it difficult to assess how their glycaemic control and diabetes respond to various interventions [2-4]. Moreover, in larger studies involving patients with higher baseline BMI, such as the Swedish Obese Subjects (SOS) study, the degree of weight loss was significantly associated with glycaemic improvement; when stratified by weight, there were no differences between restrictive and malabsorptive (i.e. 'metabolic') surgical procedures [5,6]. Insofar as the present study was not powered to evaluate that question, as the authors correctly state, no conclusions different from what we already know from much bigger datasets can be reached. Although those who defend the similarity of glycaemic improvement between individuals with a BMI $>35 \mathrm{~kg} / \mathrm{m}^{2}$ and those with a BMI $<35 \mathrm{~kg} / \mathrm{m}^{2}$ generally cite the paper on the Surgical Treatment and Medications Potentially Eradicate Diabetes Efficiently (STAMPEDE) study by Schauer et al [4], which demonstrated similar $\mathrm{HbA}_{1 \mathrm{c}}$ reductions in individuals with BMIs above and below $35 \mathrm{~kg} / \mathrm{m}^{2}$, the data presented in the supplementary appendix of this 3-year study clearly demonstrate that the daily average type 2 diabetes medication use at 36 months in the surgical group was higher in those patients with a BMI $<35 \mathrm{~kg} / \mathrm{m}^{2}$ compared with those with a higher BMI, so in this case, the 'remission rate' was significantly different between groups.

We acknowledge that nearly all bariatric surgery studies demonstrated better glycaemic control than 'usual care' diabetes treatment; we clearly believe that in moderately to morbidly obese diabetic patients, bariatric surgery is cost effective, improves diabetes control, and is likely to reduce cardiovascular risk and mortality. However, we should be cautious of extrapolating these findings to less obese patients, as prior studies indicate that the degree of weight loss is predictive of glycaemic improvement [5], of the cardiovascular risk reduction [7], and many other important endpoints including albuminuria reduction, as well as improvement of sleep apnoea and steatohepatitis, for example.

Moreover, a recent study found that despite frequent nutritional and medical visits at follow-up, at the 2-year follow-up, $80 \%$ of patients who underwent bariatric surgery presented with nutrition deficiency and five of 56 patients presented with fractures [2]. In the 3-year follow-up analysis of another study, bone and lean mass were reduced in RYGB patients [3]. The incidence of nephropathy and foot ulcers was elevated in the surgical groups (especially in the RYGB group) in the 3-year follow-up of the STAMPEDE study [4]. Other studies have reported surgical complications, including anastomotic stricture, bleeding, bowel obstruction, kidney stones and fractures [8].

In this modern era where some cardiovascular safety trials with diabetes drugs have demonstrated that these drugs provide cardiovascular protection [9, 10], we need more data on hard outcomes to better assess their efficacy and safety in very large series before advocating the widespread indication of bariatric surgery in the subpopulation of type 2 diabetic patients with a BMI $<35 \mathrm{~kg} / \mathrm{m}^{2}$, which, in absolute numbers, is the majority of diabetic patients.

Duality of interest The authors declare that there is no duality of interest associated with this manuscript.

Contribution statement All authors were responsible for drafting the article and revising it critically for important intellectual content. All authors approved the version to be published.

\section{References}

1. Cummings DE, Arterburn DE, Westbrook EO et al (2016) Gastric bypass surgery vs intensive lifestyle and medical intervention for type 2 diabetes: the CROSSROADS randomised controlled trial. Diabetologia 59:945-953

2. Ikramuddin S, Korner J, Lee WJ et al (2013) Roux-en-Y gastric bypass vs. intensive medical management for the control of type 2 diabetes, hypertension, and hyperlipidemia. The diabetes surgery study randomized clinical trial. JAMA 309:2240-2249

3. Courcoulas AP, Belle SH, Neiberg RH et al (2015) Three-year outcomes of bariatric surgery vs. lifestyle intervention for type 2 diabetes mellitus treatment. A randomized clinical trial. JAMA Surg 150:931-940

4. Schauer PR, Bhatt DL, Kirwan JP et al (2014) Bariatric surgery versus intensive medical therapy for diabetes -3 -year outcomes. N Engl J Med 370:2002-2013

5. Sjöholm K, Pajunen P, Jacobson $P$ et al (2015) Incidence and remission of type 2 diabetes in relation to degree of obesity at baseline and 2 year weight change: the Swedish Obese Subjects (SOS) study. Diabetologia 58:1448-1453

6. Sjöholm K, Sjöström E, Carlsson LMS, Peltonen M (2015) Weight change-adjusted effects of gastric bypass surgery on glucose metabolism: 2- and 10-year results from the Swedish Obese Subjects (SOS) Study. Diabetes Care 39:625-631

7. Eliasson B, Liakapoulos V, Franzén S et al (2015) Cardiovascular disease and mortality in patients with type 2 diabetes after bariatric surgery in Sweden: a nationwide, matched, observational cohort study. Lancet Diab Endocrinol 3:847-854

8. Wolfe BM, Purnell JQ, Belle SH (2013) Treating diabetes with surgery. JAMA 309:2274-2275

9. Zinman B, Wanner C, Lachin JM et al (2015) Empagliflozin, cardiovascular outcomes, and mortality in type 2 diabetes. $\mathrm{N}$ Engl J Med 373:2117-2128

10. Marso SP, Daniels GH, Brown-Frandsen K et al (2016) Liraglutide and cardiovascular outcomes in type 2 diabetes. N Engl J Med doi: 10.1056/NEJMoa1603827 